\title{
Measuring conditional cooperation: a replication study in Russia
}

\author{
Benedikt Herrmann • Christian Thöni
}

Received: 29 August 2007 / Accepted: 30 January 2008 / Published online: 10 April 2008

(C) Economic Science Association 2008

\begin{abstract}
We replicate the strategy-method experiment by Fischbacher et al. (Econ. Lett. 71:397-404, 2001) developed to measure attitudes towards cooperation in a one-shot public goods game. We collected data from 160 students at four different universities across urban and rural Russia. Using the classification proposed by Fischbacher et al. (2001) we find that the distribution of types is very similar across the four locations. The share of conditional cooperators in our Russian subject pools is comparable to the one found by Fischbacher et al. in a Swiss subject pool. However, the distribution of the other types differs from the one found in Switzerland.
\end{abstract}

Keywords Conditional cooperation · Replication · Russia

JEL Classification $\mathrm{H} 41 \cdot \mathrm{C} 91 \cdot \mathrm{C} 72$

\footnotetext{
We are grateful for the invaluable research assistance provided by Sergei Zorya, Lena Gerasimenko, Michael Belaev, Jevgenji Maslukov, Alexei Bibikov and Valerie Kowalenko. We also would like to thank the Universities of Agricultural Sciences Brijansk (Kokino), Samara (Ust-Kinel), the Belgorod State Technical Academy of Building Materials, and the All-Russian Distance Institute of Finance and Economics (ARDIFE) in Kursk for supporting us with the necessary infrastructure and Hans Kolb-Wellpappe Ltd. for the donation of cardboard screens used in the experiments. We are grateful for comments from Tim Cason, Therese Faessler, Simon Gächter, Nikhil Masters, and two anonymous referees.
}

Electronic supplementary material The online version of this article (http://dx.doi.org/10.1007/s10683-008-9197-1) contains supplementary material, which is available to authorized users.

B. Herrmann $(\bowtie)$

School of Economics, University of Nottingham, University Park, NG7 2RD Nottingham, UK e-mail: benedikt.herrmann@nottingham.ac.uk

C. Thöni

University of St. Gallen, FEW-HSG, Varnbüelstrasse 14, 9000 St. Gallen, Switzerland

e-mail: christian.thoeni@unisg.ch 


\section{Introduction}

Understanding people's attitude towards cooperation in social dilemma situations is important for a number of theoretical and policy reasons. ${ }^{1}$ To measure attitudes towards cooperation, Fischbacher et al. (2001) introduced a public goods experiment based on the strategy method. They show that roughly half of their subjects are conditional cooperators, between 20 and 30 percent are free-riders and the rest follows other patterns. ${ }^{2}$ Fischbacher et al. conducted their experiment with students in Zurich (Switzerland). This begs the question whether their results can be generalized to other subject pools.

In this study we replicate the experiment by Fischbacher et al. in Russia. Russia is interesting for testing the robustness of the Fischbacher et al. findings because strong socio-economic and cultural differences exist between Russia and Switzerland. These differences might translate into differences of measured cooperation preferences, as there is evidence that cultural backgrounds might matter for cooperation (e.g., Gächter et al. 2005; Kocher et al. 2007). We replicate the Fischbacher et al. experiment in four locations. This provides us with a strong robustness test for replicability because it allows for separating replicability within a society from potential cultural differences that might exist between societies. ${ }^{3}$

\section{Experimental design and procedures}

To measure conditional cooperation we used the exact same protocol as Fischbacher et al., which allows for the elicitation of subjects' preferences with regard to conditional cooperation. This design uses a variant of the strategy method. The subjects' task is to choose their contribution conditional on the average contribution of the other subjects in the group.

The basic decision situation is a standard one-shot linear public goods game. Subjects are randomly assigned to groups of four people. Each subject is endowed with 20 tokens, which she can either keep for herself or contribute to a "project", the public good. The payoff function is given as

$$
\pi_{i}=20-g_{i}+0.4 \sum_{j=1}^{4} g_{j},
$$

\footnotetext{
${ }^{1}$ See Gächter (2007) for a discussion and review of the literature.

${ }^{2}$ This evidence is consistent with a number of findings from experiments with different designs that do not use the strategy-method or related designs. See for instance Keser and van Winden (2000), Gächter and Thöni (2005) and Croson (2007).

${ }^{3}$ The locations where we ran our experiments also differed in their socio-economic backgrounds. Two locations are large cities and the other two are small villages, allowing for a comparison between urban and rural subject pools. This is potentially interesting because the urban-rural gap is a very profound distinction in social background widely discussed in sociology (see e.g. Durkheim 1960). Social structures in rural areas are characterized by kinship relationships and stable networks (Wirth 1964). Such structures might foster cooperation (Bowles and Gintis 2002).
} 
where the public good is equal to the sum of the contributions of all group members. Contributing a token to the public good yields a private marginal return of 0.4 and the social benefit is 1.6. Under standard assumptions all players keep their endowment for themselves.

In the experiment subjects had to choose their contribution in two variants: the unconditional and the conditional contribution. The unconditional contribution was simply a number between 0 and 20 as in the usual one-shot public goods game. For the conditional contribution subjects had to enter a "contribution table". They were shown a table of all 21 possible values of the average contribution of the other group members (rounded to integers) and were asked to enter a contribution for all cases. After all subjects had made their entries, a random device (throw of a die) selected one subject of each group to be the "conditional contributor". This subject's contribution was determined by her contribution table. For the remaining three subjects the unconditional contribution was decisive.

We went to great lengths to maximize comparability with the original experiment by Fischbacher et al. ${ }^{4}$ The only difference is that we had to run the experiments in classroom while the original experiments were conducted in a computer lab. Apart from that, exactly the same parameters and instructions were used. The experiments were conducted in a large classroom. Cardboard partitions separated the subjects from each other to avoid observer effects. The original instructions used by Fischbacher et al. were translated from German into Russian (available upon request). Another translator translated them back into German, to ensure that the Russian subjects received the same instructions. During the experiment payments were calculated in an experimental currency unit. As in the original experiment incentives were such that they well covered the opportunity cost of taking part in the experiment. ${ }^{5}$ The experiments lasted about two hours. The average earnings in Russia were $€ 2.17$.

All Russian experiments were run under the supervision of one of the authors (B.H.), who speaks Russian fluently. Local assistants supported him. He conducted the experiment according to the script from the original experiments. All participants had to read the instructions and solve a series of control questions. Subjects who showed problems in understanding the task were given assistance. The experiment did not start until all subjects had correctly solved the control questions.

All participants were undergraduate students and were recruited from large classes. We conducted the experiments in four different universities across Russia. Two of them are located in rural areas, namely in Ust-Kinel (roughly 700 miles east of Moscow) and Kokino (350 miles west of Moscow), which are small cities with roughly 5000 inhabitants each. The other two are located in urban areas, namely in the cities Kursk and Belgorod, which are both regional capitals of similar size (around 350,000 inhabitants) located south of Moscow.

\footnotetext{
${ }^{4}$ See Roth et al. (1991) for a discussion of methodological issues in conducting cross-cultural experiments.

${ }^{5}$ We questioned ten subjects who did not take part in the experiment about the hourly wages of typical student side jobs and set the exchange rate such that the average payment was slightly above the amount of money subjects could have earned during the two hours.
} 
Fig. 1 Average contribution according to the contribution table, total and for selected types

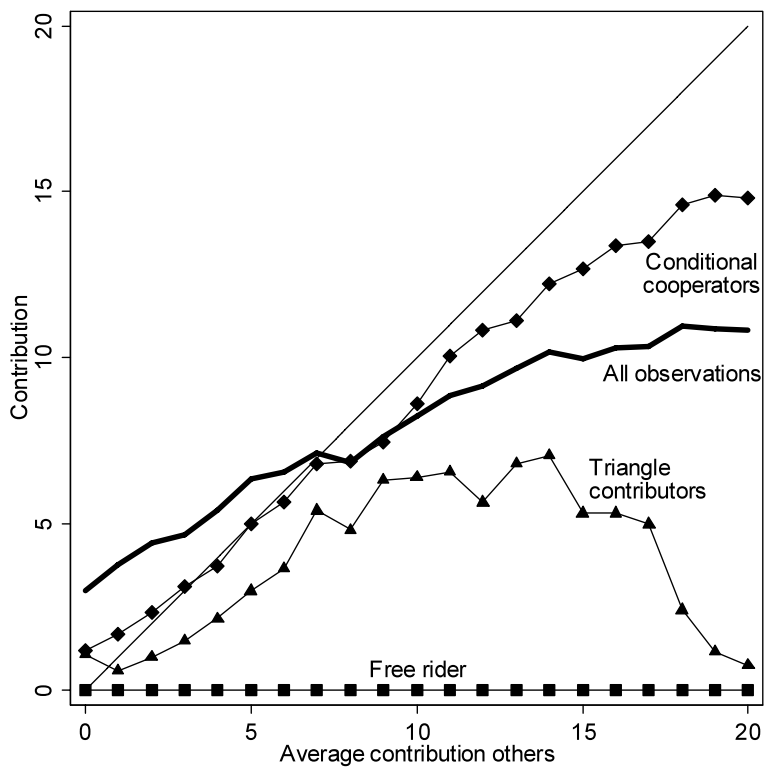

\section{Results}

As Fischbacher et al. (2001), we use the data from the contribution table to classify the subjects into four categories, namely conditional cooperators, free riders, triangle contributors, and others. All subjects whose contribution table shows either a monotonically increasing pattern or has a significant positive slope $(p<0.01$, Spearman rank correlation) are classified as conditional cooperators. A free rider is a subject who always contributes zero. Triangle contributors are subjects who have a significantly increasing pattern up to some maximum and a significantly decreasing pattern thereafter ( $p<0.01$, Spearman rank correlation). The category others consists of all remaining subjects.

Figure 1 shows the average contribution according to the contribution table of the subjects classified as conditional cooperators, free riders, and triangle contributors across all locations. The pattern of the subjects classified as conditional cooperators is very similar to the pattern observed in Fischbacher et al. The majority of conditional cooperators contribute somewhat less than the average of the other subjects' contributions.

Table 1 shows the frequency of the four types in the four locations and the whole data set. For comparison we also show the distribution of types observed by Fischbacher et al. (2001). The share of conditional cooperators varies from 47.7 to 60.0 percent (insignificantly different across the four locations, $p=0.664$, Fisher's exact test). The share of conditional cooperators is surprisingly similar to the results reported in Fischbacher et al. (2001) and a subsequent study by Fischbacher and Gächter (2006).

The share of free riders varies the most across locations (between 1.9 and 11.4 percent). However, these differences are insignificant ( $p=0.191$, Fisher's exact test). 
Table 1 Distribution of types in the four locations and the whole data set. $n$ is the number of observations. The last column shows the distribution of types observed by Fischbacher et al. (2001)

\begin{tabular}{|c|c|c|c|c|c|c|}
\hline \multirow[b]{3}{*}{$n$} & \multicolumn{2}{|l|}{ Rural } & \multicolumn{2}{|l|}{ Urban } & \multirow[t]{2}{*}{ Total } & \multirow[t]{2}{*}{ Fischbacher et al } \\
\hline & Ust-Kine & Kokino & Belgorod & Kursk & & \\
\hline & 44 & 44 & 20 & 52 & 160 & 44 \\
\hline Conditional cooperators & $47.7 \%$ & $56.8 \%$ & $60.0 \%$ & $59.6 \%$ & $55.6 \%$ & $50.0 \%$ \\
\hline Free rider & $4.5 \%$ & $11.4 \%$ & $10.0 \%$ & $1.9 \%$ & $6.3 \%$ & $29.5 \%$ \\
\hline Triangle contributors & $4.5 \%$ & $4.5 \%$ & $10.0 \%$ & $11.5 \%$ & $7.5 \%$ & $13.6 \%$ \\
\hline Others & $43.2 \%$ & $27.3 \%$ & $20.0 \%$ & $26.9 \%$ & $30.6 \%$ & $6.8 \%$ \\
\hline
\end{tabular}

The share of triangle contributors varies between 4.5 and 11.5 percent, but again the differences are insignificant ( $p=0.481$, Fisher's exact test). Both types are less frequent than reported in Fischbacher et al. The share of others is similar as well in all four subject pools ( $p=0.207$, Fisher's exact test).

To test whether a rural background of the participants has an impact on subjects' social preferences we pool the data of Ust-Kinel and Kokino as rural subject pool and Belgorod and Kursk as urban subject pool. The distribution of types is not statistically different ( $p=0.189$, Fisher's exact test).

For a finer check we ran a probit regression with the following socio-economic background variables elicited by a post-experimental questionnaire: (i) gender, (ii) age, (iii) number of siblings, (iv) relative income of parents, and (v) the size of the city where the subject spent most of her life. ${ }^{6}$ None of these variables has a significant impact on the probability of being a conditional cooperator. These results suggest that, by and large, the socio-economic environment within a society does not strongly affect people's preferences towards conditional cooperation.

\section{Concluding remarks}

The goal of this study was to replicate the experiments of Fischbacher et al. (2001) in four different locations in Russia. Our results suggest that it is important to replicate experiments in different subject pools before conclusions about generalizability can be drawn.

The two most important findings of our replication study are as follows: First, the distribution of types according to the classification proposed by Fischbacher et al. is statistically very similar across the four locations. Second, the share of conditional cooperators is comparable to the share reported in the original study. By contrast, the

\footnotetext{
${ }^{6}$ Variable (iv) has five levels, ranging from -2: Far below average, to 2: Far above average. Variable (v) is a categorical variable for the size of the city where the subjects had spent most of their live (1: Up to 2000 inhabitants, 2: 2000-10,000 inhabitants, 3: 10,000-100,000 inhabitants, 4: more than 100,000 inhabitants). In the regression we use dummies for the categories. The regression is calculated with 157 observations where the questionnaire data is complete. Variable (v) is an individual measure for our distinction between urban and rural background. The average in our rural sample is 1.7 versus 2.9 in the urban sample ( $p=$ 0.000 , Fisher's exact test).
} 
share of free riders and triangle contributors is lower than in the Zurich subject pool, whereas the fraction of others is considerably higher.

The significance of the first result is that identical procedures led to replicable findings across four different locations within the same society. In our view, this is a replication result in its own right, that is, even without comparing the results to the Fischbacher et al findings.

The second result suggests that cultural background influences attitudes towards cooperation. Given that we applied the identical protocol, instructions and control questions as in the original study and used a comparable subject pool, we are convinced that the behavioral differences are not due to differences in subjects' understanding of the task but reflect behavioral differences with respect to cooperation problems. Such differences were also identified in other studies, like e.g. Kocher et al. (2007), who investigated conditional cooperation of student subjects in Austria, Japan and USA and found the percentage of conditional cooperators to be almost twice as high in the USA (81 percent) than in the other two subject pools (44 and 42 percent). In our case the cultural background seems to have affected the nonconditional cooperators more than the conditional cooperators.

\section{References}

Bowles, S., \& Gintis, H. (2002). Social capital and community governance. The Economic Journal, 112, 419-436.

Croson, R. (2007). Theories of commitment, altruism and reciprocity: Evidence from linear public goods games. Economic Inquiry, 45, 199-216.

Durkheim, E. (1960). The division of labor in society (4th edn.). New York: Free Press.

Fischbacher, U., \& Gächter, S. (2006). Heterogeneous social preferences and the dynamics of free riding in public good. CeDEx Discussion Paper No. 2006-01, University of Nottingham.

Fischbacher, U., Gächter, S., \& Fehr, E. (2001). Are people conditionally cooperative? Evidence from public goods experiment. Economics Letters, 71, 397-404.

Gächter, S. (2007). Conditional cooperation. Behavioral regularities from the lab and the field and their policy implications. In B. S. Frey \& A. Stutzer (Eds.), Economics and psychology. A promising new cross-disciplinary field. Cambridge: MIT Press.

Gächter, S., \& Thöni, C. (2005). Social learning and voluntary cooperation among like-minded people. Journal of the European Economic Association, 3, 303-314.

Gächter, S., Herrmann, B., \& Thöni, C. (2005). Cross-cultural differences in norm enforcement. Behavioral and Brain Sciences, 28, 822-823.

Keser, C., \& van Winden, F. (2000). Conditional cooperation and voluntary contributions to public goods. Scandinavian Journal of Economics, 102, 23-39.

Kocher, M. G., Cherry, T., Kroll, S., Netzer, R. J., \& Sutter, M. (2007). Conditional cooperation on three continents. Working Papers, Faculty of Economics and Statistics, University of Innsbruck.

Roth, A. E., Prasnikar, V., Okuno-Fujiwara, M., \& Zamir, S. (1991). Bargaining and market behavior in Jerusalem, Ljubljana, Pittsburgh, and Tokyo: An experimental study. American Economic Review, $81,1068-1095$.

Wirth, L. (1964). On cities and social life. In A. J. Reiss (Ed.). Chicago: University of Chicago Press. 